\title{
Radar Coincidence Imaging under Grid Mismatch
}

\author{
Dongze Li, Xiang Li, Yongqiang Cheng, Yuliang Qin, and Hongqiang Wang \\ School of Electronic Science and Engineering, National University of Defense Technology, Changsha 410073, China \\ Correspondence should be addressed to Xiang Li; lixiang01@vip.sina.com
}

Received 6 January 2014; Accepted 28 January 2014; Published 22 April 2014

Academic Editors: I. Buciu and B. K. Gunturk

Copyright (C) 2014 Dongze Li et al. This is an open access article distributed under the Creative Commons Attribution License, which permits unrestricted use, distribution, and reproduction in any medium, provided the original work is properly cited.

\begin{abstract}
Radar coincidence imaging is an instantaneous imaging technique which does not depend on the relative motion between targets and radars. High-resolution, fine-quality images can be obtained using a single pulse either for stationary targets or for complexly maneuvering ones. There are two image-reconstruction algorithms used for radar coincidence imaging, that is, the correlation method and the parameterized method. In comparison with the former, the parameterized method can achieve much higher resolution but is seriously sensitive to grid mismatch. In the presence of grid mismatch, neither of the two algorithms can obtain recognizable high-resolution images. The above problem largely limits the applicability of radar coincidence imaging in actual imaging scenes where grid mismatch generally exists. This paper proposes a joint correlation-parameterization algorithm, which uses the correlation method to estimate the grid-mismatch error and then iteratively modifies the results of the parameterized method. The proposed algorithm can achieve high resolution with fine imagery quality under the grid mismatch. Examples are provided to illustrate the improvement of the proposed method.
\end{abstract}

\section{Introduction}

Radar coincidence imaging is developed as the extension of classical coincidence imaging in microwave radar systems [1]. Classical coincidence imaging, which is realized in thermal optical systems, is a method to nonlocally image an object with high resolution by producing sharply fluctuating signals in the imaging plane $[1,2]$. Similarly, the essential principle of radar coincidence imaging is to produce time-space independent signals in the detecting area. Then radar signals at different positions have mutually independent waveforms. Illuminated by such signals, target-scattering centers within a radar beam will also reflect echoes of independent waveforms associated with their respective positions. Therefore, the echo component of each scattering center can be extracted from the receiving signal and then correlated to their respective positions. Consequently, scattering centers within a beam can be resolved and the spatial distribution of the target scattering centers can be obtained. Obviously, it is quite different from the radar imaging techniques based on the range-Doppler $(\mathrm{RD})$ principle, where scattering centers are resolved based on the analysis of time-delay and Doppler frequency [3].
Because of the different imaging principles, the radar coincidence imaging technique has two advantages over most of the RD imaging methods. Firstly, radar coincidence imaging does not depend on the aspect-angle integration or the Doppler gradient to achieve high azimuth resolution. Thus, it does not require relative motion between radars and targets and can obtain images of the targets which remain stationary with respect to radars. Furthermore, radar coincidence imaging can achieve high-resolution using a single pulse. The extremely short imaging time, which is shorter than a pulse width, considerably decreases the impact of the noncooperative motion to imagery quality. Therefore, radar coincidence imaging can obtain high-resolution, finequality images either for stationary targets or for the ones in complex maneuvers.

In radar coincidence imaging, the target area needs to be discretized to a grid and target-scattering centers are assumed to be located at the grid points. If scattering centers are located off the grid points, then the grid mismatch yields. There are two main image-reconstruction methods used in radar coincidence imaging. One is the correlation method with lower resolution. The other is the parameterized method 


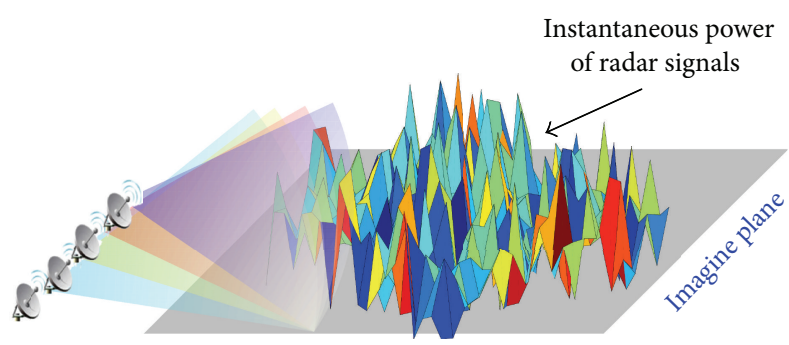

(a)

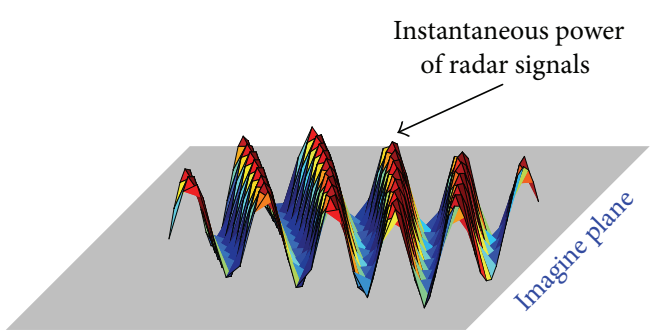

(b)

FIGURE 1: (a) The spatial distribution of time-space detecting signals. (b) The spatial distribution produced by coherent transmitted signals.

which can produce much higher resolution but is too sensitive under grid mismatch to give recognizable target images. Furthermore, current algorithms that are applicable to solve the sensitivity to basis mismatch are unfortunately ineffective for the grid mismatch in radar coincidence imaging [4, 5]. This limitation seriously affects the applicability of the imaging method in actual scenarios where the grid mismatch generally exists. This paper proposes a joint correlationparameterization method for image reconstruction which can achieve both high resolution and good imagery quality under grid mismatch.

The paper is organized as follows. Section 2 is devoted to the basic principles of radar coincidence imaging. Section 3 analyzes the impact of grid mismatch and proposes the joint correlation-parameterization method for image reconstruction. Section 4 concludes the work.

\section{Basic Principles}

Coherent signals, which are widely employed by most of the imaging radars, generally produce detecting signals that show significant spatial correlation, as shown in Figure 1(b). However, the essence of radar coincidence imaging is to produce time-space independent radar signals in the detecting area. In other words, the detecting signals at different instants or in different positions are independent of each other. As shown in Figure 1(a), at an arbitrary instant, the time-space independent detecting signals fluctuate sharply in the imaging plane and its instantaneous spatial distribution presents remarkable variety.

Generally, the target location can be firstly estimated based on the detection and localization techniques [6,7], which is defined as the center of the target area. Then a local coordinate system is established in the target area center, as shown in Figure 2. The target area is discretized to a grid consisting of $L$ small rectangles of uniform size and shape. Each small rectangle is the grid cell and is approximated by its own center. Thus, target-scattering centers are actually assumed to be initially located at the grid-cell centers. The discrete target area is expressed as $I=\left\{\mathbf{r}_{1}, \mathbf{r}_{2}, \ldots, \mathbf{r}_{L}\right\}$, where $\mathbf{r}_{l}$ is the position vector of the $l$ th grid cell center. There are $N$ transmitters and a receiver, whose position vectors are $\mathbf{R}_{n}$ and $\mathbf{R}_{r}$, respectively. The transmitted signal of the $n$th transmitter is denoted as $S t_{n}(t)$ and the receiving signal is $\operatorname{Sr}(t)$. The detecting signals distributed in the target area are labeled as

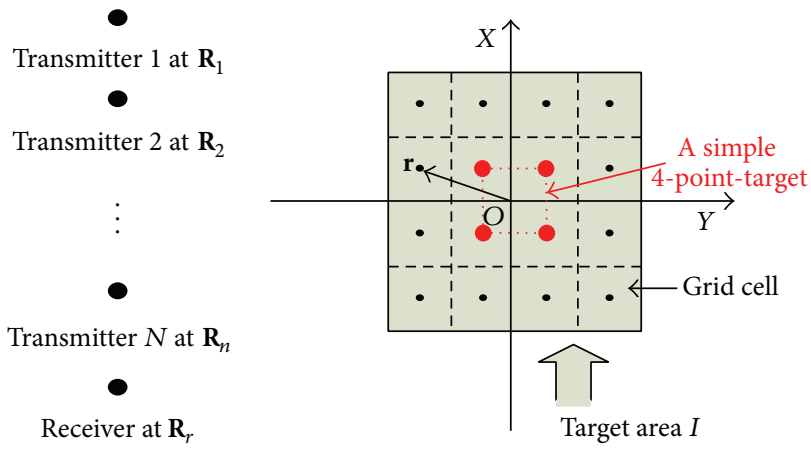

FIGURE 2: Geometry of the target area grid.

$S_{I}(\mathbf{r}, t)$, where $\mathbf{r}$ is the position vector of an arbitrary grid cell within $I$.

The ideal transmitted signals for radar coincidence imaging are supposed to be group-orthogonal and timeindependent as denoted in (1), which can produce the time-space independent $S_{I}(\mathbf{r}, t)$ via single transmitting [1]. Consider

$$
\begin{aligned}
R_{T}\left(n_{1}, n_{2} ; t_{1}, t_{2}\right) & =\int S t_{n_{1}}\left(t-t_{1}\right) S t_{n_{2}}\left(t-t_{2}\right) d t \\
& =\delta\left(n_{1}-n_{2}, t_{1}-t_{2}\right),
\end{aligned}
$$

where $S t_{n}(t)=\operatorname{rect}\left(t / T_{p}\right) \cdot s t_{n}(t), s t_{n}(t)$ is the envelope function and $T_{p}$ is the pulse width. If the antenna number is more than 2, for example, $N=5$, the detecting signal produced by such transmitted signals will show approximate time-space independence [1], expressed as follows:

$$
\begin{aligned}
R_{I}\left(\mathbf{r}, \mathbf{r}^{\prime} ; \tau, \tau^{\prime}\right) & =\int S_{I}(\mathbf{r}, t-\tau) S_{I}^{*}\left(\mathbf{r}^{\prime}, t-\tau^{\prime}\right) d t \\
& \sim N \delta\left(\mathbf{r}-\mathbf{r}^{\prime}, \tau^{\prime}-\tau\right) .
\end{aligned}
$$

Then, we express the receiving signal as the superposition of the detecting signals. Consider

$$
S r(t)=\sum_{l=1}^{L} \sigma_{l} S_{I}\left(\mathbf{r}_{l}, t-\frac{\left|\mathbf{r}_{l}-\mathbf{R}_{r}\right|}{c}\right)
$$

where $\sigma_{l}$ is the scattering coefficient of the scattering center located at the $l$ th grid cell and for the grid cell without 
target-scattering center $\sigma_{l}=0$. For the sake of simplicity, the coincidence imaging formulism needs a reference signal [1], which herein can be simply structured using $S_{I}(\mathbf{r}, t)$ as follows:

$$
S(\mathbf{r}, t)=S_{I}\left(\mathbf{r}, t-\frac{\left|\mathbf{r}-\mathbf{R}_{r}\right|}{c}\right) .
$$

Consequently, (3) becomes

$$
S r(t)=\sum_{l=1}^{L} \sigma_{l} S\left(\mathbf{r}_{l}, t\right) .
$$

Then, the scattering coefficient of an arbitrary grid cell $\mathbf{r}_{x}$ can be explicitly obtained via the correlation between the receiving signal and $S\left(\mathbf{r}_{x}, t\right)$,

$$
\begin{aligned}
\int S r(t) S^{*}\left(\mathbf{r}_{x}, t\right) d t= & \int\left(\sum_{l=1}^{L} \sigma_{l} \cdot S_{I}\left(\mathbf{r}_{l}, t-\frac{\left|\mathbf{r}_{l}-\mathbf{R}_{r}\right|}{c}\right)\right) \\
& \cdot S_{I}^{*}\left(\mathbf{r}_{x}, t-\frac{\left|\mathbf{r}_{x}-\mathbf{R}_{r}\right|}{c}\right) d t \\
= & \sum_{l=1}^{L} \sigma_{l} \cdot R_{I}\left(\mathbf{r}_{l}, \mathbf{r}_{x} ; \frac{\left|\mathbf{r}_{l}-\mathbf{R}_{r}\right|}{c}, \frac{\left|\mathbf{r}_{x}-\mathbf{R}_{r}\right|}{c}\right) \\
\sim & \sum_{l=1}^{L} \sigma_{l} \cdot N \delta\left(\mathbf{r}_{l}-\mathbf{r}_{x}\right) \\
= & N \cdot \sigma_{x} .
\end{aligned}
$$

That is,

$$
\sigma_{x} \sim \frac{1}{N} \int \operatorname{Sr}(t) S^{*}\left(\mathbf{r}_{x}, t\right) d t
$$

Note that the detecting signal $S_{I}(\mathbf{r}, t)$ or $S(\mathbf{r}, t)$ can be computed based on the known transmitted waveforms. Thus, the scattering coefficient of each grid cell can be derived via the correlation between the receiving signal and the reference signal. Therefore, the target scene can be recovered via conducting the correlation in (7) for the whole target area grid. This method to reconstruct target images is defined as the correlation method.

The excellent point-to-point relationship in (7) means a high resolution, but it requires that the transmitted signals have the perfect time-independence as presented in (1). Unfortunately, microwave transmitted signals are far from this requirement and their time-independent degree is inadequate to achieve the high resolution represented in (7) via the correlation method [1]. Therefore, to improve the resolution of radar coincidence imaging, the parameterized method is employed which is less constrained by the signal timeindependence. This method uses the relationship between the receiving signal and the reference signal, as shown in (5), to structure a radar coincidence imaging equation, given as follows:

$$
\begin{gathered}
\mathbf{S r}=\mathbf{S} \cdot \boldsymbol{\sigma}, \\
{\left[\begin{array}{c}
S r\left(t_{1}\right) \\
S r\left(t_{2}\right) \\
\vdots \\
S r\left(t_{K}\right)
\end{array}\right]=\left[\begin{array}{cccc}
S\left(\mathbf{r}_{1}, t_{1}\right) & S\left(\mathbf{r}_{2}, t_{1}\right) & \cdots & S\left(\mathbf{r}_{L}, t_{1}\right) \\
S\left(\mathbf{r}_{1}, t_{2}\right) & S\left(\mathbf{r}_{2}, t_{2}\right) & \cdots & S\left(\mathbf{r}_{L}, t_{2}\right) \\
\vdots & \vdots & \cdots & \vdots \\
S\left(\mathbf{r}_{1}, t_{K}\right) & S\left(\mathbf{r}_{2}, t_{K}\right) & \cdots & S\left(\mathbf{r}_{L}, t_{K}\right)
\end{array}\right] \cdot\left[\begin{array}{c}
\sigma_{1} \\
\sigma_{2} \\
\vdots \\
\sigma_{L}
\end{array}\right],}
\end{gathered}
$$

where $K=L, \mathbf{S}$ is the reference signal matrix, $\mathbf{S r}$ is the vector of the receiving signal, and $\boldsymbol{\sigma}$ is the unknown vector of the scattering coefficient. The columns and the rows of $\mathbf{S}$ basically represent the detecting signals in different positions and at different instants, respectively. Hence, the incoherent property of $\mathbf{S}$ is basically determined by the timespace independence of the detecting signals. Therefore, the independent characteristic of the detecting signal will ensure a full-rank S. Consequently, the target scattering-coefficient vector can be uniquely recovered as $\boldsymbol{\sigma}=\mathbf{S}^{-1} \cdot \mathbf{S r}$.

\section{Impact of the Grid Mismatch and Image Reconstruction}

The imaging equation presented in (8) is derived under the grid-match condition. Generally, regardless of how finely the target area is gridded, target-scattering centers may not lie in the grid-cell centers. Assume that there exists the grid mismatch. The scattering-center position vector actually is $\mathbf{r}_{l}+\Delta \mathbf{r}_{l}$ instead of the assumed $\mathbf{r}_{l}$ for the $l$ th grid cell. Thus, the actual receiving signal is $S r(t)=\sum_{l=1}^{L} \sigma_{l} S\left(\mathbf{r}_{l}+\Delta \mathbf{r}_{l}, t\right)$. Consequently, the imaging equation should be $\mathbf{S r}=\left(\mathbf{S}+\boldsymbol{\varepsilon}_{s}\right)$. $\boldsymbol{\sigma}$, where $\boldsymbol{\varepsilon}_{s}$ is caused by the difference between $S\left(\mathbf{r}_{l}, t\right)$ and $S\left(\mathbf{r}_{l}+\Delta \mathbf{r}_{l}, t\right)$. As $\boldsymbol{\varepsilon}_{s}$ and $\boldsymbol{\sigma}$ are both unknown variables, the above imaging equation can be simplified as

$$
\mathbf{S r}=\mathbf{S} \cdot \boldsymbol{\sigma}+\boldsymbol{\varepsilon}_{m}
$$

where $\boldsymbol{\varepsilon}_{m}=\boldsymbol{\varepsilon}_{s} \cdot \boldsymbol{\sigma}$ and denotes the modeling error caused by grid mismatch. Then (9) represents the well-known linear signal model. Thus, the actual scattering-coefficient vector should be derived as $\boldsymbol{\sigma}=\mathbf{S}^{-1} \cdot\left(\mathbf{S r}-\boldsymbol{\varepsilon}_{m}\right)$. The previous solution derived from (8) is relabeled as $\widehat{\boldsymbol{\sigma}}$. That is, $\mathbf{S r}=\mathbf{S} \cdot \widehat{\boldsymbol{\sigma}}$, where $\boldsymbol{\varepsilon}_{m}$ is neglected because of the grid-match assumption. Certainly, the imaging quality of the parameterized method will be decreased due to the modeling error.

To illustrate the grid-mismatch impact, we give an example to show how the position bias affects the modeling error and how the modeling error affects the imaging quality. In the quantitative manner, the imaging quality discussed here is firstly indicated by the relative imaging error, expressed as $\|\hat{\boldsymbol{\sigma}}-\boldsymbol{\sigma}\| /\|\boldsymbol{\sigma}\|$. Then the modeling error is normalized as the signal-error-ratio, denoted as $\zeta=20 \log _{10}\|\mathbf{S r}\| /\left\|\boldsymbol{\varepsilon}_{m}\right\|$. The position bias is defined as $\sum_{l=1}^{L}\left|\Delta \mathbf{r}_{l}\right| / l_{c}$, where $l_{c}$ is the grid-cell perimeter.

The example employs an $N$-transmitter 1-receiver array, consisting of 5 antennas. The arrangement of the antennas and the target is shown in Figure 3. The target area is 


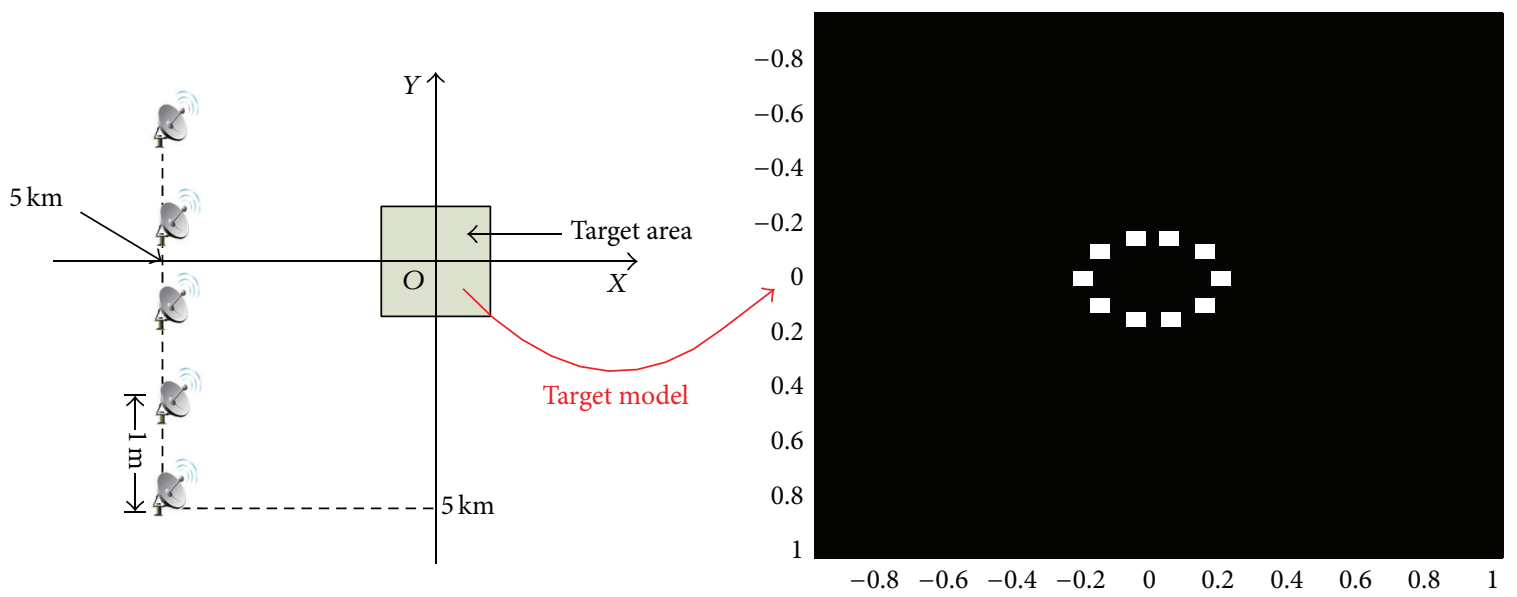

FIGURE 3: The arrangement of antennas and the target model.

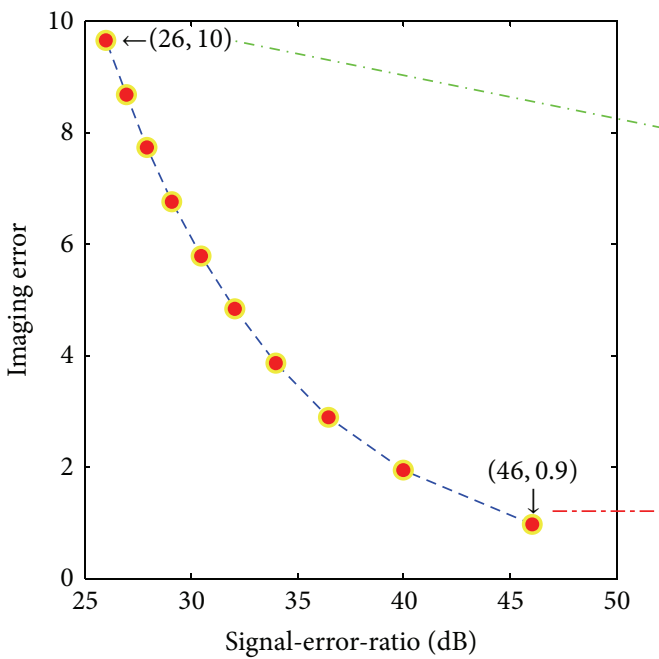

(a)

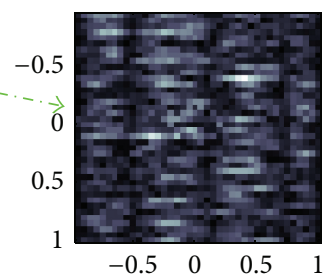

(b)

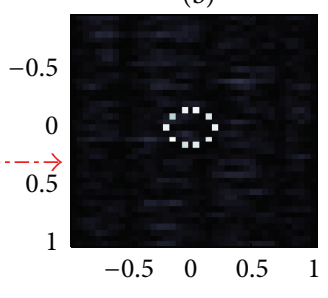

(c)

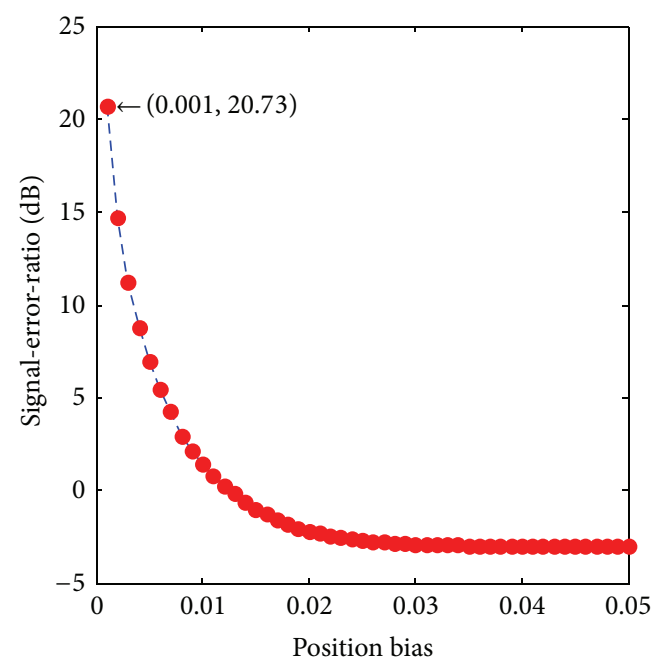

(d)

FIGURE 4: The grid-mismatch impact to imaging quality of the parameterized method. (a) The imaging error versus $\zeta$. (b) The imaging result when $\zeta=26 \mathrm{~dB}$. (c) The imaging result when $\zeta=46 \mathrm{~dB}$. (d) The signal-error-ratio versus the position bias.

$2 \mathrm{~m} \times 2 \mathrm{~m}$ and is discretized to $40 \times 40$ grid cells, each of which is $5 \mathrm{~cm} \times 5 \mathrm{~cm}$. The group-orthogonal and timeindependent transmitted signals herein are generated by modulating the sinusoid signals on amplitude via mutually independent Gaussian noises. The carrier frequency is $9.5 \mathrm{GHz}$, the pulse width is $50 \mu \mathrm{s}$, the bandwidth is $1 \mathrm{GHz}$, and the sampling frequency is $2 \mathrm{GHz}$. The results are shown in Figure 4.

In this example, the target image in Figure 4(b) is badly blurred beyond reorganization when $\zeta=26 \mathrm{~dB}$. The target image in Figure 4(c) is recognizable when the imaging error is 0.9 , which requires that $\zeta$ should not be lower than $46 \mathrm{~dB}$. It demonstrates that the parameterized method has a low tolerance to the modeling error. A recognizable image with a small imaging error requires a small modeling error or a high signal-error-ratio. Then the question is "will the position bias generally produce a high-level signal-error-ratio to guarantee low imaging error?" Unfortunately, as shown in Figure 3(d), even a very small position bias of 0.001 in this example can cause a large modeling error, which only provides $20 \mathrm{~dB}$ single-error-ratio. Moreover, the position bias less than 0.02 will generate a negative $\zeta$. It shows that the modeling error is seriously sensitive to the position bias. The position bias of 0.04 can cause $-4 \mathrm{~dB}$ signal-error-ratio in radar coincidence imaging equation, with no mention of a much larger bias in practice. In this case, modeling error almost overwhelms the receiving signal in the imaging equation. The experiment indicates that the recovery, simply depending on the imaging equation, might impossibly provide a good estimation under such a poor signal-error-ratio condition.

Consider another example to compare the correlation method with the parameterized method under grid mismatch. In the example, target images will be reconstructed via the two methods with and without the grid mismatch. Herein, the grid mismatch leads to $\zeta=-4 \mathrm{~dB}$. Imaging results are shown in Figure 5. 


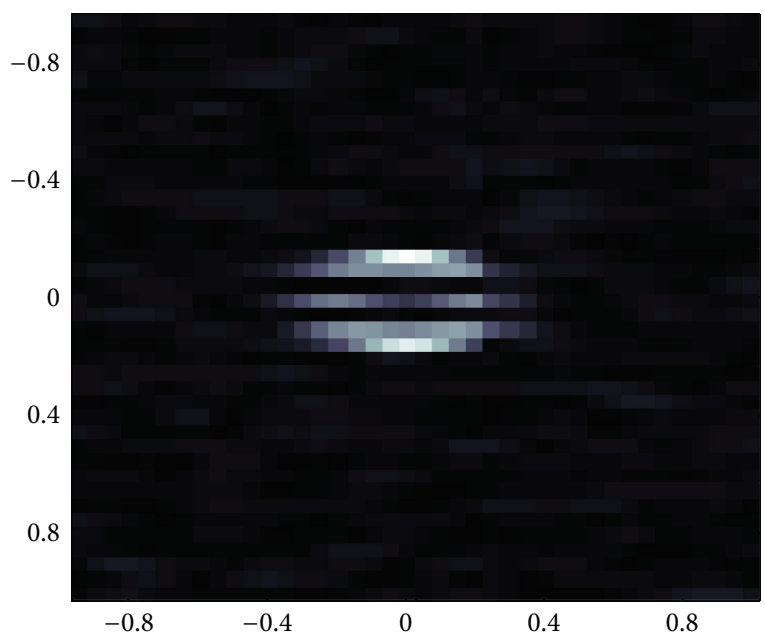

(a)

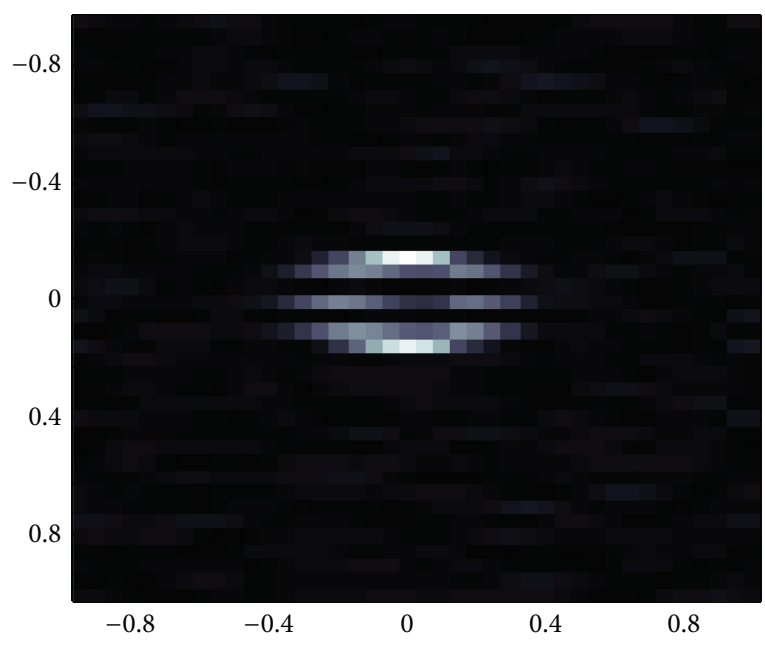

(c)

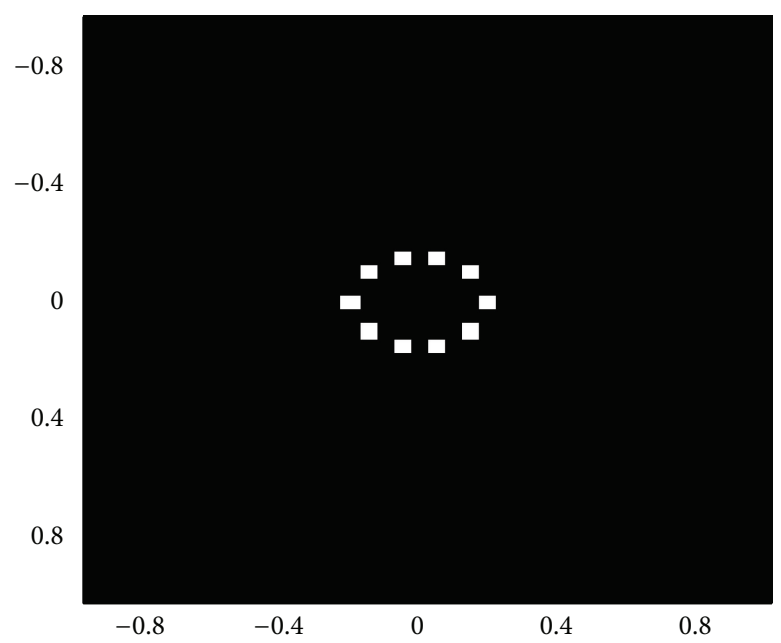

(b)

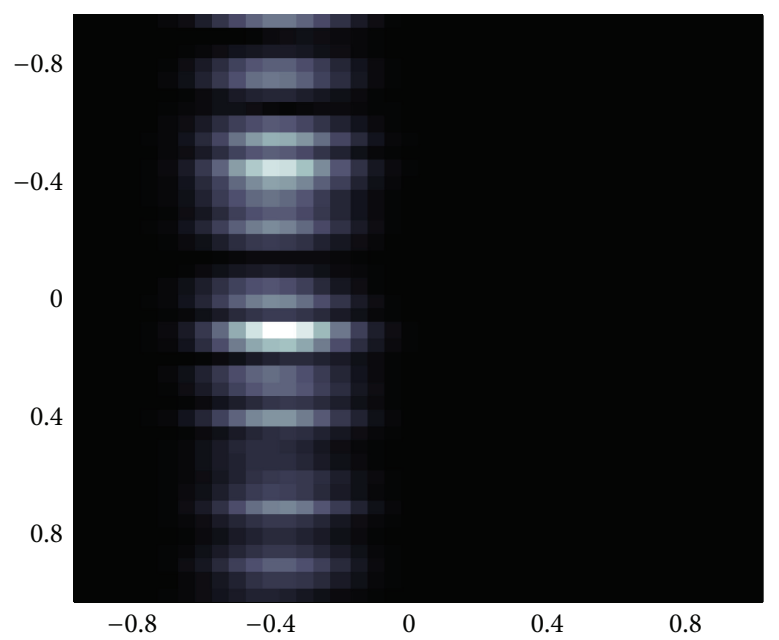

(d)

FIGURE 5: The imaging results recovered by different methods. (a) The correlation-method result without grid mismatch. (b) The parameterized-method result without grid mismatch. (c) The correlation-method result when $\zeta=-4 \mathrm{~dB}$. (d) The parameterized-method result when $\zeta=-4 \mathrm{~dB}$.

As shown in Figure 5, the parameterized method provides higher resolution than the correlation method in the case of no grid mismatch. On the other side, when $\zeta=$ $-4 \mathrm{~dB}$, the imaging quality of the correlation method is almost unchanged but the parameterized-method result gets degraded and almost gives no information on the target shape. It is obvious that the correlation method is less sensitive to the modeling error than the parameterized method but has much lower resolution.

In conclusion, neither of the two image-reconstruction methods can achieve both high resolution and good imaging quality in the presence of grid mismatch. According to their respective limitations, there are two ways to solve the image reconstruction under grid mismatch. One way is to improve the resolution of the correlation method. The other one is to decrease the grid-mismatch impact in the parameterized method. As stated previously, however, microwave signal does not have adequate time-independence in nature, resulting in a limited resolution. Thus, the latter might be a possible way to solve the problem of grid mismatch.

A direct idea to decrease grid-mismatch impact in the parameterized method is to estimate the modeling error. Note that the modeling error is relevant to the scattering center number, scattering intensity, and the position-bias level of all scattering centers. If there is no prior information of the target shape, the modeling error will be quite difficult to estimate. Then for the targets that are unknown in advance, the obtainable shape information could be sought from the imaging results of the two reconstruction methods. Thus, a possible source to provide the desired knowledge would be the correlation-method result, considering the seriously distorted images of the parameterized method caused by grid mismatch.

The knowledge that can be used by the imaging equation should be quantitative and precise. However, the result of the correlation method, as observed in Figure 5, presents a 


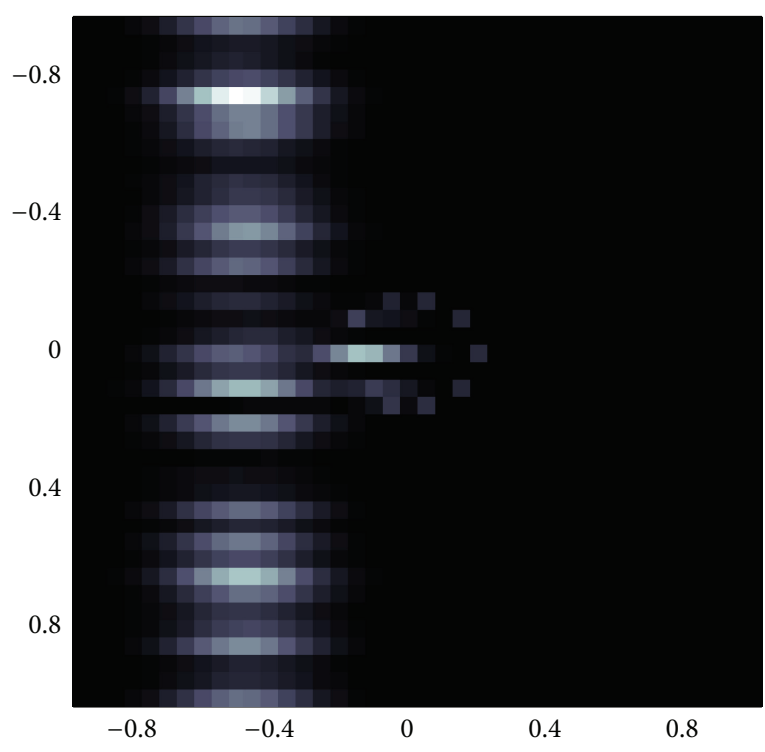

(a)

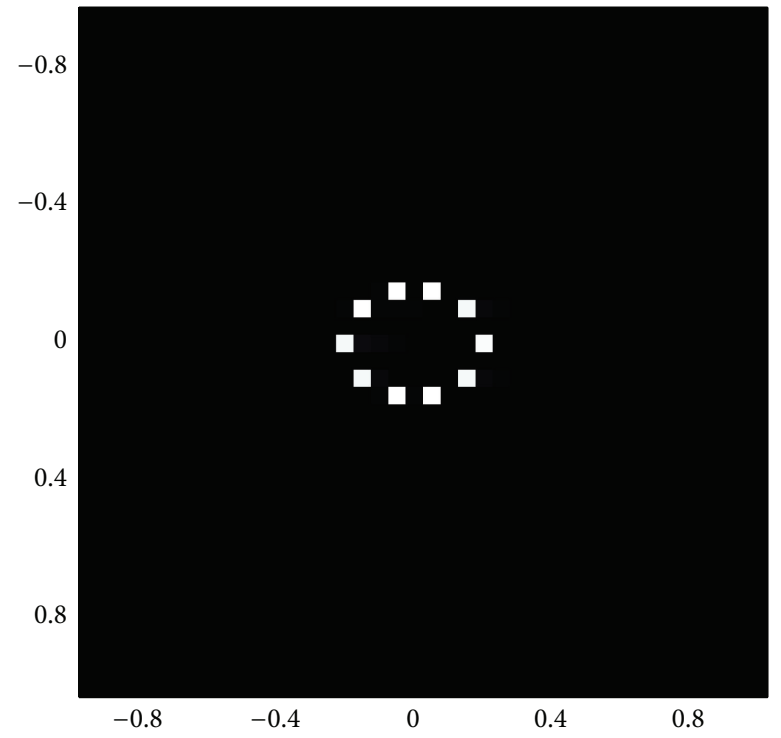

(b)

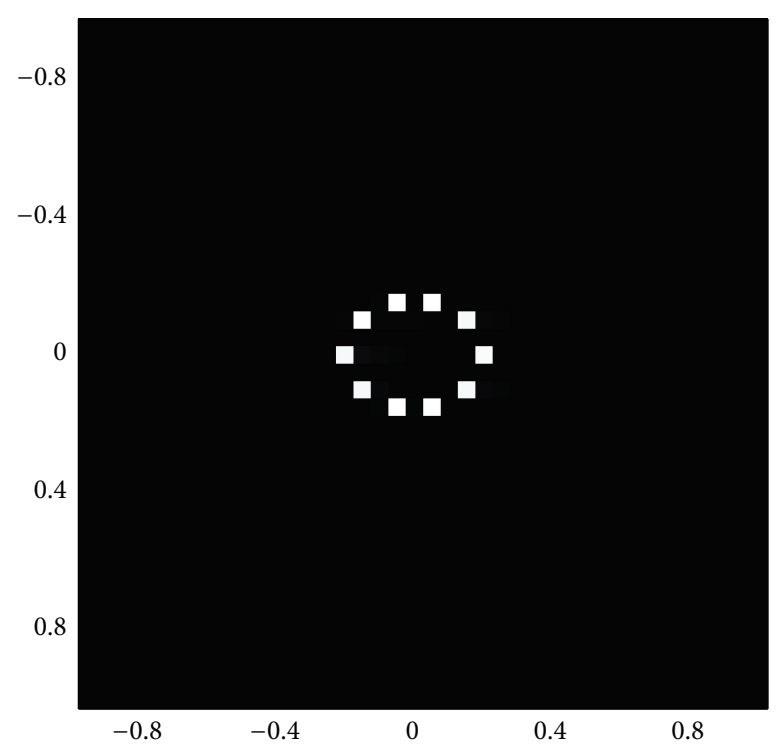

(c)

FiguRE 6: The imaging results of the joint correlation-parameter method. (a) The imaging result of the first iteration. (b) The imaging result of the second iteration. (c) The imaging result of the third iteration.

TABLE 1: The flow of the joint correlation-parameterization method.

\begin{tabular}{ll}
\hline Step 1 & Set the iteration step maximum $T_{\max }$ and the stop limitation $\eta, k=0$. \\
Step 2 & Discretize the target area and compute the reference signal. \\
Step 3 & Obtain the scattering-coefficient vector $\widehat{\boldsymbol{\sigma}}_{c}$ using the correlation method. \\
Step 4 & Set a threshold $\sigma_{T}$ to determine $\Lambda=\left\{l_{1}, l_{2}, \ldots, l_{M}\right\}$, where $\widehat{\boldsymbol{\sigma}}_{c}\left(l_{m}\right)<\sigma_{T}$. \\
Step 5 & Compose the coincidence imaging equation. Obtain the initial estimation $\widehat{\boldsymbol{\sigma}}^{(k)}$ using the parameterized method. Set Sr $\mathbf{r}^{(k)}=\mathbf{S r}$. \\
Step 6 & Compute the inverse matrix of the reference matrix $\mathbf{S}$, and then according to $\Lambda$ obtain $\mathbf{S}_{\Lambda}^{-1}=\left[\boldsymbol{\alpha}_{l_{1}}, \boldsymbol{\alpha}_{l_{2}}, \ldots, \boldsymbol{\alpha}_{l_{M}}\right]^{T}$. \\
Step 7 & Derive $\widehat{\boldsymbol{\sigma}}_{\Lambda}^{(k)}=\left[\widehat{\sigma}_{l_{1}}^{(k)}, \widehat{\sigma}_{l_{2}}^{(k)}, \ldots, \widehat{\sigma}_{l_{M}}^{(k)}\right]^{T}$. \\
Step 8 & Compute the modeling noise $\widehat{\boldsymbol{\varepsilon}}_{m}^{(k)}=\mathbf{S}_{\Lambda}^{\dagger} \widehat{\boldsymbol{\sigma}}_{\Lambda}^{(k)}, k=k+1$. \\
Step 9 & Update the scattering-coefficient vector as $\widehat{\boldsymbol{\sigma}}^{(k)}=\mathbf{S}^{-1}\left(\mathbf{S r}^{(k-1)}-\widehat{\boldsymbol{\varepsilon}}_{m}^{(k-1)}\right)$. \\
Step 10 & Stop if $\left\|\widehat{\boldsymbol{\sigma}}^{(k)}-\widehat{\boldsymbol{\sigma}}^{(k-1)}\right\| \leq \eta$ or $k \geq T_{\max }$. Otherwise $\mathbf{S r}^{(k)}=\mathbf{S r}^{(k-1)}-\widehat{\boldsymbol{\varepsilon}}_{m}^{(k)}$, and go to Step 7. \\
\hline
\end{tabular}


blurry target shape. The image cannot precisely indicate the target scattering-center location or provide the exact value of the scattering coefficient. Nonetheless, it indeed provides valuable information. That is, the correlation-method result can denote part of the grid cells which do not contain targetscattering centers. In Figures 5(a) and 5(c), only a minority of image patches, which constitute the target contour, have large values. By contrast, a majority of other image patches have far too small values, which implies the corresponding grid cells have no scattering centers. Hence, these corresponding elements in the scattering-coefficient vector are estimated to be zero. Such quantitative knowledge will be helpful for the estimation of the modeling error. Detailed implementation of image reconstruction using the idea will be described in the following paragraphs.

Firstly, still under the grid-match assumption, we estimate the target scattering-coefficient vector via the two reconstruction methods: $\widehat{\boldsymbol{\sigma}}_{c}$ is derived via the correlation method and $\widehat{\boldsymbol{\sigma}}$ is obtained via the parameterized method. According to $\mathbf{S r}=\mathbf{S} \cdot \widehat{\boldsymbol{\sigma}}$ and (9), we have the following relationship:

$$
\mathbf{S} \cdot \widehat{\boldsymbol{\sigma}}-\mathbf{S} \cdot \boldsymbol{\sigma}=\boldsymbol{\varepsilon}_{m} \longrightarrow \widehat{\boldsymbol{\sigma}}-\boldsymbol{\sigma}=\mathbf{S}^{-1} \boldsymbol{\varepsilon}_{m}
$$

where $\mathbf{S}^{-1}$ is written as $\left[\boldsymbol{\alpha}_{1}, \boldsymbol{\alpha}_{2}, \ldots, \boldsymbol{\alpha}_{L}\right]^{T}, \boldsymbol{\alpha}_{l}$ being the $l$ th rowvector of $\mathbf{S}^{-1}$. The next step is to select the grid cells without scattering centers according to $\widehat{\boldsymbol{\sigma}}_{c}$. In comparison with the average value of $\widehat{\boldsymbol{\sigma}}_{c}$, part of the elements in $\widehat{\boldsymbol{\sigma}}_{c}$, which have far too small values, are determined to be 0 . The index of these zero-elements is denoted as $\Lambda=\left\{l_{1}, l_{2}, \ldots, l_{M}\right\}$; that is, $\sigma_{l_{m}}=$ $0, l_{m} \in \Lambda$. According to $\Lambda$, we define $\mathbf{S}_{\Lambda}^{-1}=\left[\boldsymbol{\alpha}_{l_{1}}, \boldsymbol{\alpha}_{l_{2}}, \ldots, \boldsymbol{\alpha}_{l_{M}}\right]^{T}$, $\widehat{\boldsymbol{\sigma}}_{\Lambda}=\left[\widehat{\sigma}_{l_{1}}, \widehat{\sigma}_{l_{2}}, \ldots, \widehat{\sigma}_{l_{M}}\right]^{T}$, and $\boldsymbol{\sigma}_{\Lambda}=\left[\sigma_{l_{1}}, \sigma_{l_{2}}, \ldots, \sigma_{l_{M}}\right]^{T}$. Based on (10), we have

$$
\widehat{\boldsymbol{\sigma}}_{\Lambda}-\boldsymbol{\sigma}_{\Lambda}=\mathbf{S}_{\Lambda}^{-1} \cdot \boldsymbol{\varepsilon}_{m}
$$

As $\boldsymbol{\sigma}_{\Lambda}=\mathbf{0}$, finally $\widehat{\boldsymbol{\sigma}}_{\Lambda}=\mathbf{S}_{\Lambda}^{-1} \cdot \boldsymbol{\varepsilon}_{p}$. Therefore, the modeling noise is estimated as

$$
\widehat{\boldsymbol{\varepsilon}}_{m}=\mathbf{S}_{\Lambda}^{\dagger} \widehat{\boldsymbol{\sigma}}_{\Lambda},
$$

where $\mathbf{S}_{\Lambda}^{\dagger}$ is the pseudoinverse matrix of $\mathbf{S}_{\Lambda}^{-1}$. By substituting $\widehat{\boldsymbol{\varepsilon}}_{m}$ to (9), the new target scattering-coefficient vector obtained is

$$
\widehat{\boldsymbol{\sigma}}^{(1)}=\mathbf{S}^{-1}\left(\mathbf{S} \mathbf{r}-\widehat{\boldsymbol{\varepsilon}}_{m}\right)
$$

If the result remains unsatisfactory, we can repeat the computation of (12) and (13) using the new $\widehat{\boldsymbol{\sigma}}^{(1)}$ and $\widehat{\boldsymbol{\varepsilon}}_{m}$. That is, update the receiving signal as $\mathbf{S r}^{(1)}=\mathbf{S r}-\widehat{\boldsymbol{\varepsilon}}_{m}$. Then derive $\widehat{\boldsymbol{\sigma}}_{\Lambda}^{(1)}$ according to $\Lambda$ and update the modeling error as $\widehat{\boldsymbol{\varepsilon}}_{m}^{(1)}=\mathbf{S}_{\Lambda}^{\dagger} \widehat{\boldsymbol{\sigma}}_{\Lambda}^{(1)}$. Finally, the estimation is updated as $\widehat{\boldsymbol{\sigma}}^{(2)}=$ $\mathbf{S}^{-1}\left(\mathbf{S} \mathbf{r}^{(1)}-\widehat{\boldsymbol{\varepsilon}}_{m}^{(1)}\right)$. The update can be performed iteratively until the result is satisfactory. This image-reconstruction method using both the correlation method and the parameterized method is defined as the joint correlation-parameterization method. The detailed steps are given in Table 1.

To examine the correlation-parameterization method, target images are reconstructed according to the iteration process of Table 1, where the grid mismatch is the same as that in Figure 5(d). The imaging results are given in Figure 6.

As shown in Figure 6, a high-quality target image can be obtained through three iterations. The result of the first iteration has presented the target contour, though the contour is light and the imaging error remains remarkable. After the second iteration, the imaging quality is largely improved. By contrast, the imaging quality of Figure 6(b) is much better than the result in Figure 5(d). The third iteration maintains the good imagery quality. Therefore, two iterations actually give a high-resolution and fine-quality target image. It indicates that the joint correlation-parameterization method satisfies the requirement for both the resolution and the robustness in the presence of the modeling noise caused by grid mismatch.

\section{Conclusions}

Radar coincidence imaging can achieve excellent highresolution target images on the condition of grid match, but the imagery quality gets degraded beyond recognition in the presence of the modeling error caused by grid mismatch. Therefore, the paper proposes the joint correlationparameterization method for image reconstruction. The proposed algorithm iteratively modifies the parameterizedmethod result with the estimated modeling error, which is obtained based on the correlation-method result. Consequently, the grid-mismatch impact on the imaging quality is considerably reduced. The example shows that the joint correlation-parameterization method can achieve high resolution and maintain the robustness under grid mismatch.

\section{Conflict of Interests}

The authors declare that there is no conflict of interests regarding the publication of this paper.

\section{References}

[1] D. Li, X. Li, Y. Cheng, Y. Qin, and H. Wang, "Radar coincidence imaging: an instantaneous imaging technique with stochastic signals," IEEE Transactions on Geoscience and Remote Sensing, vol. 52, no. 4, 2014.

[2] A. Gatti, E. Brambilla, M. Bache, and L. A. Lugiato, "Ghost imaging with thermal light: comparing entanglement and classical correlation," Physical Review Letters, vol. 93, no. 9, Article ID 093602, 2004.

[3] Y. Shih, "Quantum imaging," IEEE Journal on Selected Topics in Quantum Electronics, vol. 13, no. 4, pp. 1016-1030, 2007.

[4] Y. Chi, L. L. Scharf, A. Pezeshki, and A. R. Calderbank, "Sensitivity to basis mismatch in compressed sensing," IEEE Transactions on Signal Processing, vol. 59, no. 5, pp. 2182-2195, 2011.

[5] M. A. Herman and T. Strohmer, "General deviants: an analysis of perturbations in compressed sensing," IEEE Journal on Selected Topics in Signal Processing, vol. 4, no. 2, pp. 342-349, 2010. 
[6] P. Z. Peebles, Radar Principles, John Wiley \& Sons, New York, NY, USA, 1998.

[7] J. S. Thorp, "Optimal tracking of maneuvering targets," IEEE Transactions on Aerospace and Electronic Systems, vol. 9, no. 4, pp. 512-519, 1973. 

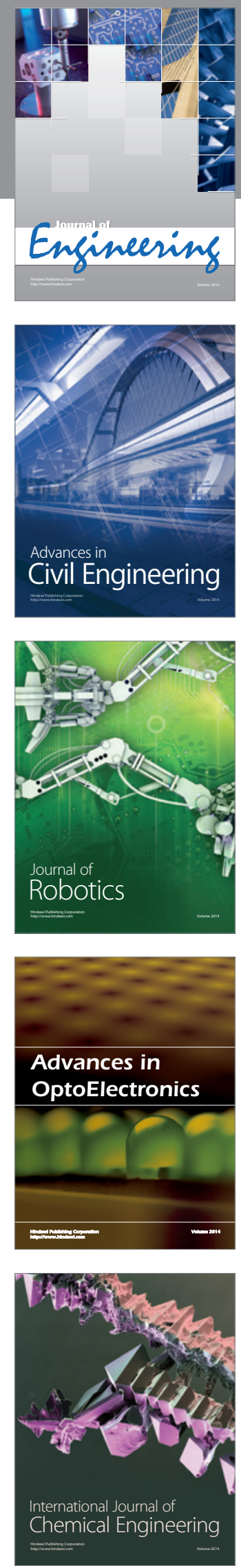

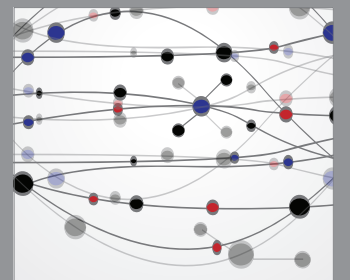

The Scientific World Journal
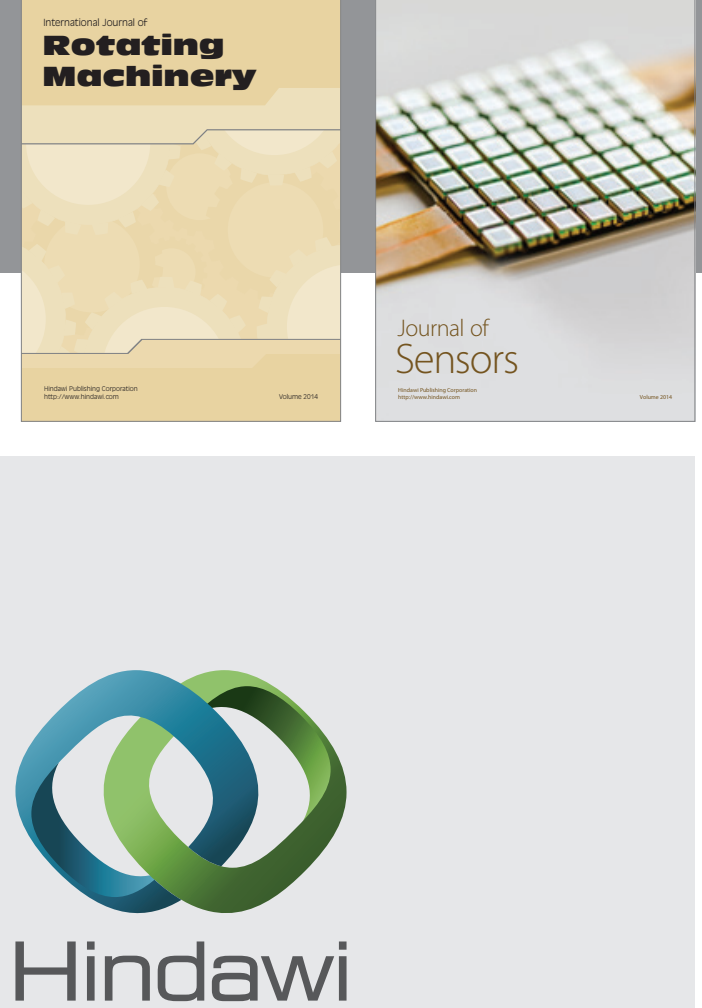

Submit your manuscripts at http://www.hindawi.com
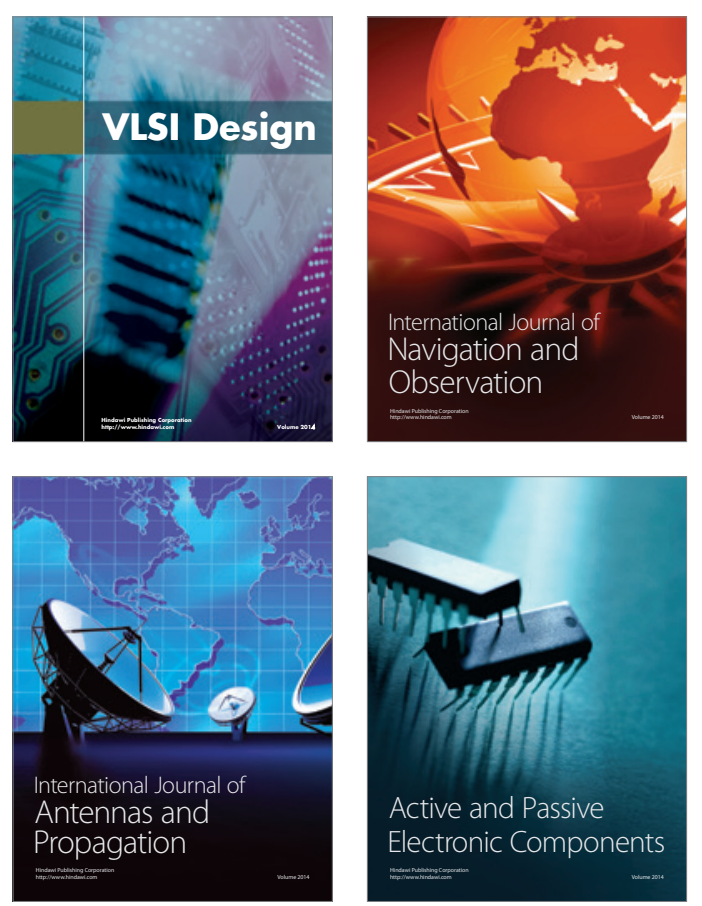
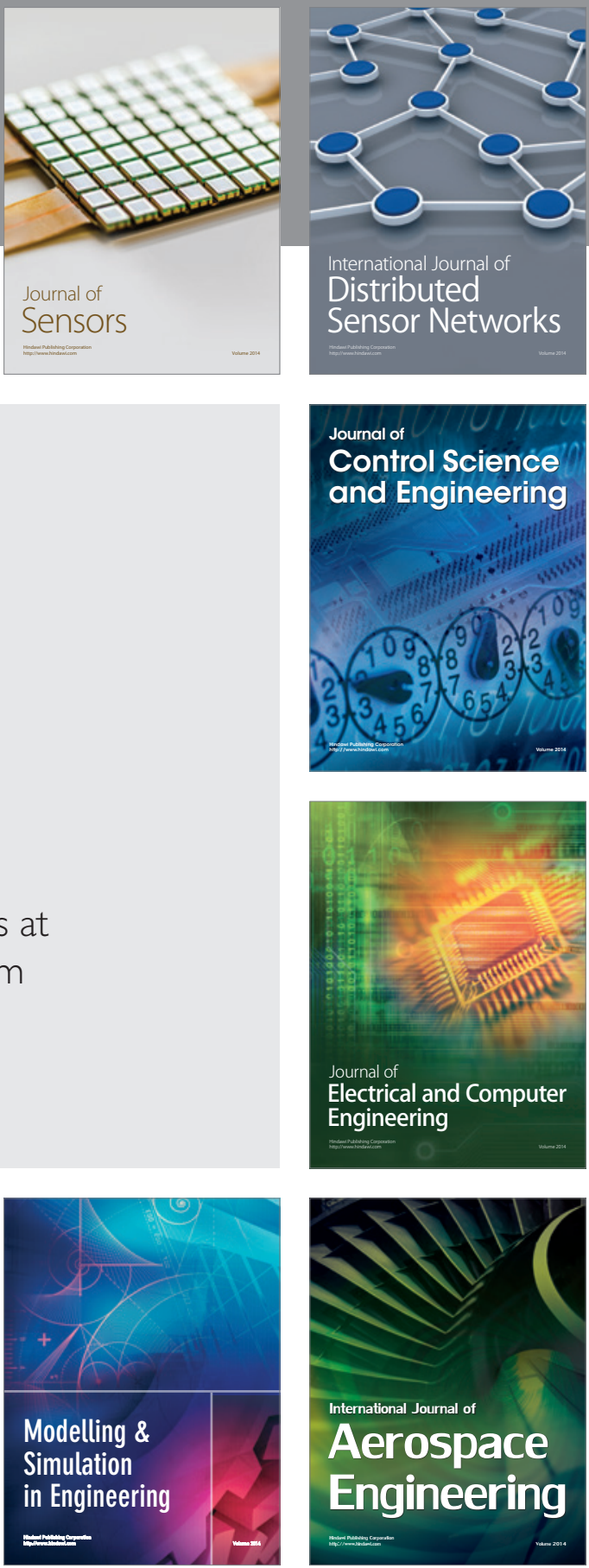

Journal of

Control Science

and Engineering
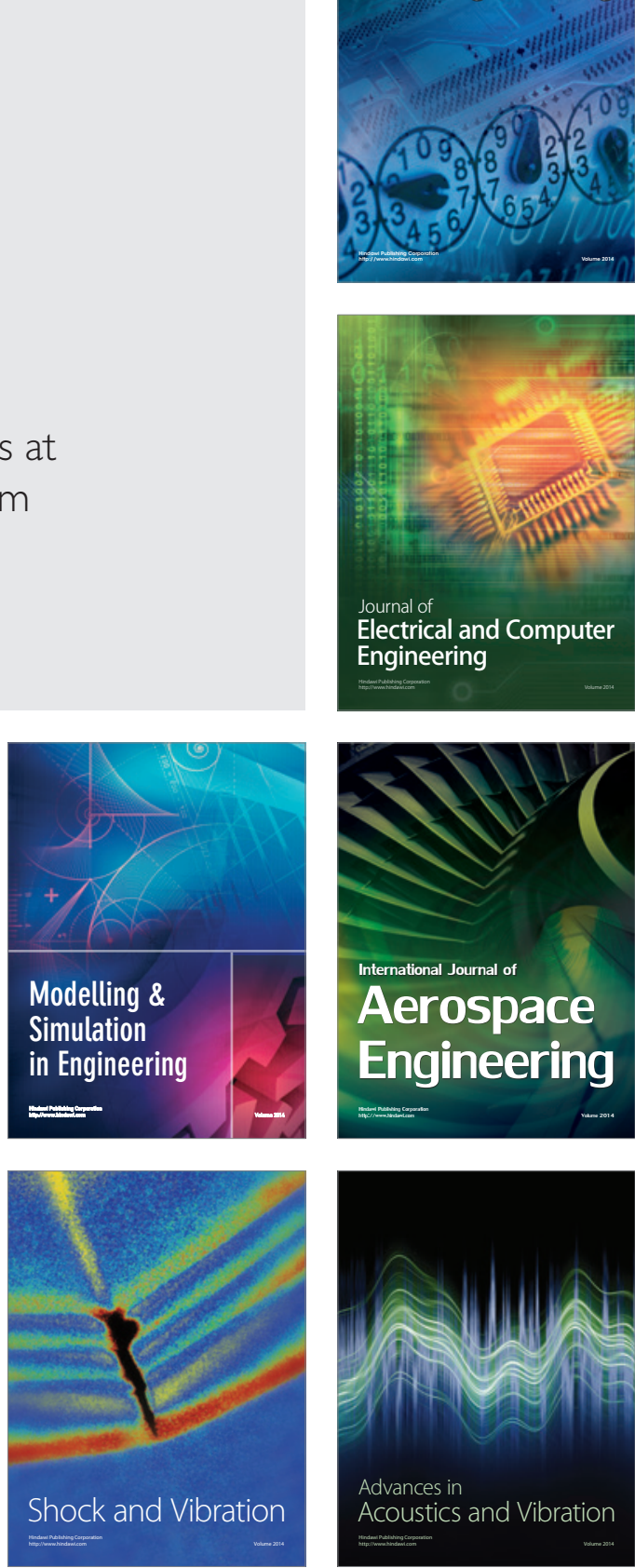